\title{
Flow separation over a backward-facing ramp with and without a vortex generator
}

\author{
Siddhesh Shinde, Suyash Tandon $†$ Kevin Maki ${ }^{\ddagger}$ and Eric Johnsen ${ }^{\S}$ \\ University of Michigan, Ann Arbor, MI, 48109, USA
}

\begin{abstract}
Large Eddy Simulation (LES) and Unsteady Reynolds-Averaged Navier-Stokes (URANS) calculations are performed for flow over a $25^{\circ}$ backward-facing ramp with and without a passive vortex generator (VG). The objective of this work is to determine the influence of the VG on the separated flow region. The LES study is performed with a synthetic time-varying turbulent inflow boundary condition whereas a mean turbulent velocity inlet is used for URANS. The wall layer is resolved in the LES and these results are used as a baseline to evaluate the accuracy of the URANS calculations. A dynamic one equation model is used for LES while the $k-\omega$ SST model is used for URANS. Upstream of the ramp edge the URANS simulations lack the near wall turbulence structures observed in LES due to the synthetic inflow. Therefore URANS calculations overpredict the reattachment length as compared to LES with and without the VG. Based on the turbulent kinetic energy profiles the separation tends to induce more turbulence away from the wall and decrease it close to the wall. The effect of the VG is to entrain momentum from the mean flow to the near wall region, therefore reducing the reattachment length. Proper Orthogonal Decomposition (POD) is used to identify the dominant modes in separation region with and without the VG.
\end{abstract}

\section{Introduction}

Many practical engineering applications exhibit flow separation and reattachment. The phenomenon of flow separation and the stages that lead to it have been studied in great detail in the past. Yet, investigating the flow characteristics in the separated region and reattachment region is still an area of active research. The capability to simulate the separation and reattachment process is critical to understand the strategies we need to control the separation. Controlling boundary layer separation using passive and active methods is an ongoing research topic. The ability to control boundary layer separation effectively can be leveraged to reduce drag and increase lift on airfoils at low Reynolds number, ${ }^{9}$ to reduce losses in subsonic diffuser ${ }^{2}$ and to reduce after-body drag in aircraft fuselage. ${ }^{3}$

A great amount of work has been done in understanding flow separation like Stratford's work on developing the criterion for turbulent boundary layer separation on a flat plate ${ }^{24}$ (1959). Work done by Simpson et al. ${ }^{21}$ on measurements of adverse pressure gradient flows revealed that the log law is maintained until close to separation. More recently, Direct Numerical Simulation (DNS) of separating turbulent boundary layer done by $\mathrm{Na}$ and Moin ${ }^{19}$ revealed stark similarities in the kinetic energy budget in the separated region to that of a plane mixing layer. Along with studying the underlying universal nature of the boundary layer in separated regions, the subsequent reattachment and recovery of the boundary layer have been investigated with equal interest. In the review on recovery of a boundary layer, Smits et al. ${ }^{22}$ report that the recovery process initiates from the wall and grows outwards in the shear layer. However, Alving et al. ${ }^{1}$ present a

\footnotetext{
*Graduate Student, Department of Mechanical Engineering, 1231 Beal Ave., 2043 Walter E. Lay Automotive Laboratory, Ann Arbor, MI 48109-2133, AIAA member.

${ }^{\dagger}$ Graduate Student, Department of Mechanical Engineering, 1231 Beal Ave., 2043 Walter E. Lay Automotive Laboratory, Ann Arbor, MI 48109-2133, AIAA member.

$¥$ Assistant Professor, Naval Architecture and Marine Engineering, 210 NAME Building, 2600 Draper Drive, Ann Arbor, MI 48109-2145, AIAA member.

$\S$ Assistant Professor, Department of Mechanical Engineering, 1231 Beal Ave., 2043 Walter E. Lay Automotive Laboratory, Ann Arbor, MI 48109-2133, AIAA member.
} 
contrary view observed while studying mild separation of a turbulent boundary layer in which the recovery process is initiated in the outer layer.

Conventionally, passive vortex generators (VGs) that are approximately of the size $(h)$ of the boundary layer thickness $(\delta)$ have been used to control flow separation. When an attached boundary layer encounters an adverse pressure gradient it expends its momentum against the pressure gradient to remain attached to the surface. The point at which the velocity gradient at the wall becomes zero the flow can no longer remain attached and separates from the surface. The manner in which passive VGs help in controlling flow separation is that they energize the boundary layer by entraining high momentum fluid from the mean flow to the near wall region as seen in figure $1 .{ }^{17}$ This helps to keep the boundary layer attached to the surface. Passive VGs can be used effectively if the point of separation is well defined in the flow domain. They were first used by Taylor ${ }^{25}$ in 1947. Since then many configurations like the cylinders, the Wheeler doublets, wish-bones etc. ${ }^{16}$ have been studied. Traditionally, VGs with $h \sim \delta$ have been used in aerospace applications for localized flow control over short streamwise distances. ${ }^{17}$ However, these VGs have a greater device drag associated with them on account of their large size. Therefore, it is expected that an optimal configuration and shape can be developed for low-profile VGs for applications where the separation point is fixed. $\operatorname{Lin}^{17}$ has documented the significant milestones in the study of low-profile passive VGs in controlling boundary layer separation. In the exploratory study done by Rao and Kariya ${ }^{20}$ they suggest that the submerged VGs with $h / \delta \leqslant 0.65$ have better performance because of the low device drag. From ${ }^{17,20}$ and several others it is clear the effectiveness of the VG in controlling boundary layer separation depends on the size of the VG $(h)$ relative to the boundary layer thickness $(\delta)$, the spanwise spacing between the VGs $(\Delta z / h)$ and the streamwise distance between the VG trailing edge and the line of separation $(\Delta x / h)$.

The aim of this study is twofold. First, we seek to understand the effect of a VG $(h / \delta \sim 0.6)$ on the separation and reattachment process when the flow separates from the edge of the ramp. Second, we seek to evaluate the performance the $k-\omega$ SST URANS model in predicting the flow characteristics. To achieve the first objective a wall resolved LES of flow over a $25^{\circ}$ backward facing ramp is performed using the dynamicone equation eddy viscosity model ${ }^{6}$ with and without a passive VG. To achieve the second objective URANS calculations of the same flow are performed and the results are compared with LES data.

While several studies have been done in the past to control separation using passive VGs they are experimental in nature and a high-fidelity numerical substantiation on this geometry is lacking. The novelty of this work is that we perform a wall resolved LES of the flow (on our fine mesh). Also we use a synthetic time varying inflow boundary condition to replicate a spatially evolving flat plate boundary layer. The geometry, mesh specification and numerical setup of our simulations is explained in section II followed by a discussion of the results in section III.

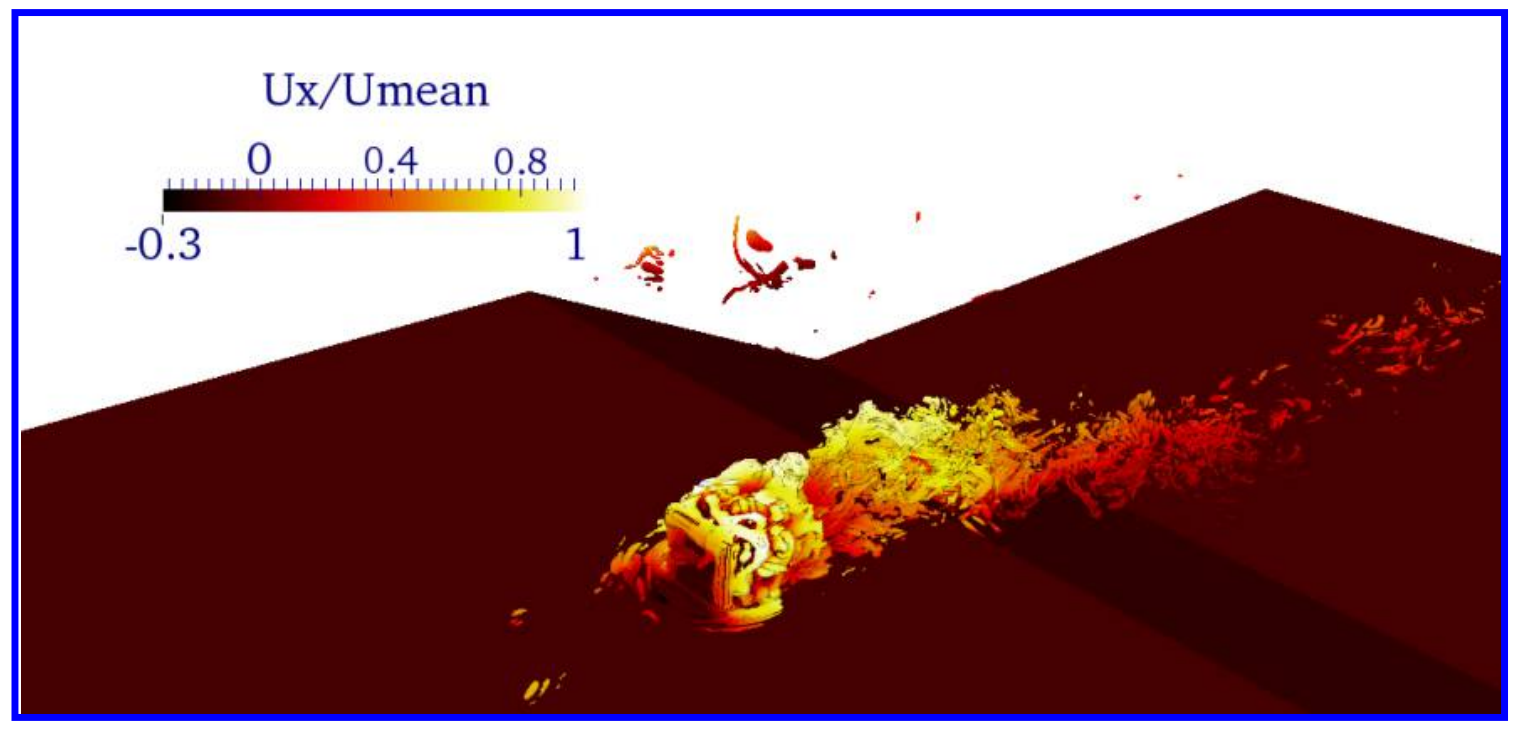

Figure 1. Iso-surfaces of Q criterion colored with normalized x-component of velocity. 


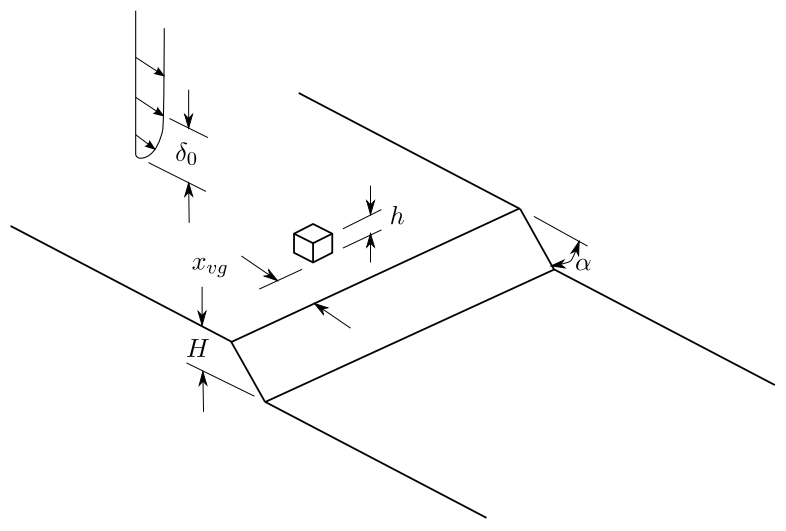

Figure 2. Schematic of the setup.

\section{Computational setup}

\section{II.A. Geometry}

A $25^{\circ}$ ramp is used to study flow separation from the edge of the ramp. The ramp height is denoted as $H$ as seen in figure 2. The dimension upstream of the ramp surface is $6 H$ and downstream ramp surface is $6 H$ in length. The Reynolds number $\left(R e_{H}\right)$ based on the free-stream velocity and the ramp height $(H)$ is 28,956 . The width of the domain is $4.7 \mathrm{H}$. The boundary layer thickness $\left(\delta_{o}\right)$ at the inlet of the domain is $\delta_{o} / H=0.394$. We choose to work with this geometry primarily because of two reasons. First, it is a canonical geometry with a fixed point of separation which is ideal for passive flow control. Second, the ramp angle and height are the only two parameters required to define the geometry.

According to prior work done on separation control using passive VGs, vane-type VGs with $h / \delta_{o} \sim$ 0.2 are found to be most effective when placed at a streamwise distance of $5 h-10 h$ from the point of separation. ${ }^{16,17}$ However, to determine the optimal VG configuration for the backward-facing ramp it is important to understand the effect of the VG on the flow. Therefore, we consider a single cube placed in the plane of symmetry of the domain. The height $(h)$ of the VG is $h / \delta_{o}=0.64$. The VG is placed at a distance of $x_{\mathrm{vg}}=3 h$ upstream from the ramp edge. The choice of using the cube as a VG for optimal flow control was motivated by the fact that it has a single length dimension associated with it which helps us to reduce the dimensions of the parameter space. We will explore the parameter space with different cube heights $\left(h_{\text {cube }}\right)$ and upstream VG locations $\left(x_{\mathrm{vg}}\right)$ in our future work.

\section{II.B. Mesh specification}

To numerically investigate flow over a backward facing ramp using LES, we designed the mesh to have high resolution near the wall region. In wall coordinates the average grid spacing is $\Delta_{y}^{+} \sim 1$ in the wall normal direction and in streamwise and spanwise directions the resolution was $\Delta_{x}^{+}, \Delta_{z}^{+} \sim 10$. To reduce simulation time we mapped our results from the coarse mesh to the fine mesh as an initial condition. The coarse grid had approximately 5 million cells, while the fine mesh had around 20 million cells.

Table 1. Grid specification for the case without VG.

\begin{tabular}{lccc} 
Property & Coarse mesh & Medium mesh & Fine mesh \\
\hline Total number of cells $\left(\times 10^{6}\right)$ & 5.1 & 14.9 & 19.9 \\
$\Delta_{y}^{+*}$ in the refined region & 10 & 5 & 1 \\
${ }^{*}$ The "+" indicates the dimensionless grid spacing in wall coordinates. $\Delta^{+}=\frac{\Delta u_{\tau}}{\nu}$ \\
where $\Delta$ is the grid spacing in physical dimensions, $u_{\tau}$ is the friction velocity at the \\
wall and $\nu$ is the kinematic viscosity of the fluid.
\end{tabular}




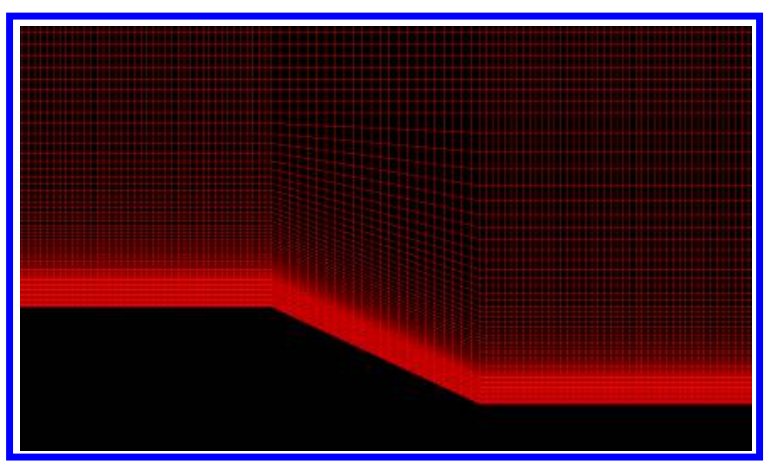

(a) without VG

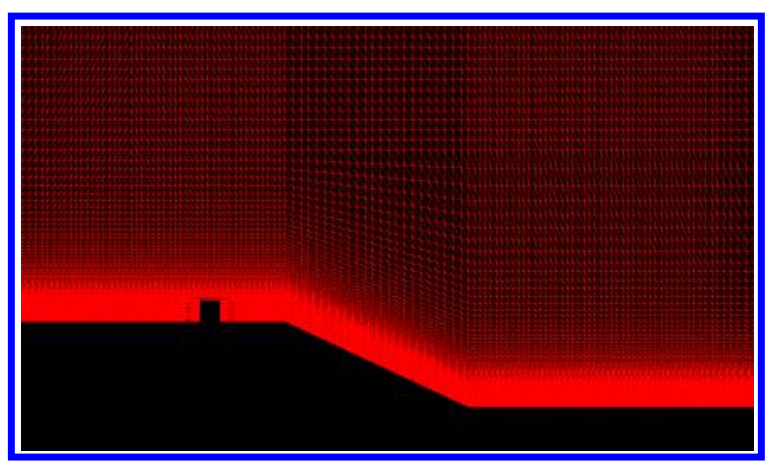

(b) with VG

Figure 3. Front view of the center plane of the mesh.

Table 2. Grid specification for the case with VG.

\begin{tabular}{lccc} 
Property & Coarse mesh & Medium mesh & Fine mesh \\
\hline Total number of cells $\left(\times 10^{6}\right)$ & 5.1 & 14.9 & 19.9 \\
Number of cells/cube height & 10 & 20 & 30 \\
$\Delta_{y}^{+}$in the refined region & 10 & 5 & 1
\end{tabular}

The background mesh is refined locally such that the region $4.9 H_{\text {ramp }}$ upstream of ramp edge, over the ramp and $4.3 H_{\text {ramp }}$ downstream of the ramp has uniform resolution in streamwise and spanswise flow directions while a small grading in refinement exists in the wall normal direction. In the spanwise direction we used a finer grid spacing near the center plane and the region adjacent to the sides of the domain to capture any effects on the flow influenced by the side walls. The vortical structures around the cube are not affected by width $(W)$ of the domain as long as $W / h_{\text {cube }}>5 .^{7}$ From LES results of flow past a cube placed in a channel ${ }^{13}$ it was found that the horse-shoe vortex formed in front of the cube extends to a distance of $1.6 h_{\text {cube }}$. Dandois et al. ${ }^{5}$ (2007) performed DNS of active separation control of flow over a backward-facing smooth ramp. In this study they reported that for $y / H_{\text {ramp }}>2$ the flow is quasi-potential in nature. Based on these facts from prior work we chose to refine the mesh locally as seen in figure 3 .

\section{II.C. Numerical setup}

In the LES study, the filtered incompressible Navier-Stokes equations are solved using the dynamic-one equation eddy viscosity model as described by Germano et al. ${ }^{6,10}$ In the URANS study, the Reynolds Averaged Navier-Stokes equations are solved using the $k-\omega$ SST model. ${ }^{27}$ A second-order linear upwind stabilized transport scheme is used for the convective term and a second-order central scheme with explicit correction for the diffusion term. A second-order implicit backward scheme is used for time marching. We used the OpenFOAM CFD package to perform the numerical simulations.

The simulation is run for a total time of $900 \mathrm{H} / U_{\infty}$. For the LES calculations the statistical averaging is performed from $t=450 H / U_{\infty}$. We allow 60 "flow throughs" before statistically averaging the data to avoid contamination of results due to the initial transient. The time required for one "flow through" is defined as time required for the flow to move past the downstream surface of the ramp with an average velocity $\left(U_{\text {avg }} \approx 0.8 U_{\infty}\right)$. The boundary conditions for the simulation are no-slip on the bottom wall, ramp and the surface of the cube. A slip wall boundary condition is used on the side and top walls. A zero-gradient boundary condition is specified at the outlet. In the URANS study a constant mean turbulent boundary layer profile given by Spalding ${ }^{23}(1961)$ is used as a inflow boundary condition. In the LES study a time varying turbulent boundary layer condition is used at the inlet as described in section II.C.1

\section{II.C.1. Inflow boundary condition}

To simulate a spatially evolving turbulent boundary layer (SETBL) on the upstream ramp surface a synthetic time varying velocity profile is prescribed at the inlet. The turbulent boundary layer is obtained by adding 
fluctuations on a mean velocity profile. The mean velocity profile given by Spalding ${ }^{23}$ (1961) is used in our method.

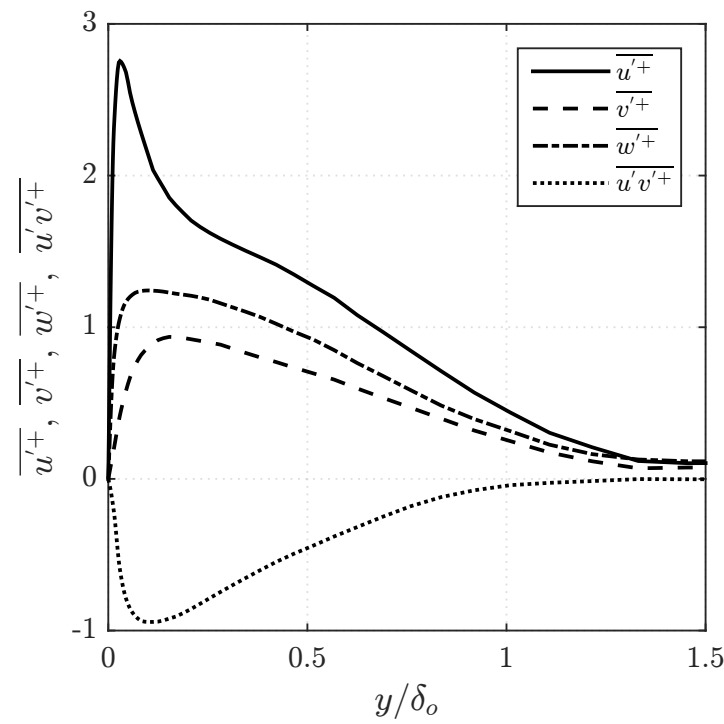

Figure 4. Turbulent intensities as a function of outer coordinate $y / \delta_{o}$ at the inlet boundary.

In order to generate the time varying fluctuations we generated a random initial solenoidal field in a cube as described in Appendix A of Johnsen et al. ${ }^{8}$ (2010). Since the fluctuation field obtained by this procedure is homogenous and isotropic whereas the turbulent wall-bounded flow over the ramp is non-homogenous in the wall-normal direction, we modify the fluctuation field according to the procedure outlined by $\mathrm{Le}^{14}$ (1995). The Reynolds stress profiles at the inlet match well with those of Wu et al. ${ }^{28}$ as seen in figure 4 . He performed DNS of flow over a backward-facing step. As described by $\mathrm{Le}^{14}$ the SETBL requires a transition length of $x / \delta_{o} \approx 10$. We tested this method on various grid resolutions. The boundary layer growth profiles and turbulent intensities agree well with DNS results of Wu et al. ${ }^{28}$

The inlet boundary condition is varied at a time step given by the grid velocity, $\Delta t=\Delta_{\mathrm{xavg}} / U_{\infty}$, where $\Delta_{\text {xavg }}$ is the average grid spacing in the streamwise direction. For all the results presented in this paper, the simulations were performed with an inlet boundary layer thickness of $\delta_{o} / H=0.394$.

\section{Results and discussion}

\section{III.A. Mean and intergral quantities}

In this section we will compare the URANS predictions with our wall resolved LES results for the cases with and without the VG.

In figure 5 we observe the variation of time averaged skin friction along the bottom wall in the center plane of the domain. In the case without the VG in figure $5(\mathrm{a})$ at $x / H_{\mathrm{ramp}}=0$ the sharp drop in the skin friction marks the point of flow separation along ramp top edge. The skin friction is negative from $x / H_{\text {ramp }}=0$ to 2.1 because of the recirculation bubble on the ramp. The sharp spike at $x / H_{\text {ramp }}=2.1$ is due to near stagnation conditions at the bottom edge of the ramp. Along the bottom wall of the ramp between $x / H_{\text {ramp }}=2.1$ and 3 the negative velocity close to the wall increases followed by a recovery of the velocity to a positive value near the wall at $x / H_{\text {ramp }}=5$. Beyond this point the boundary layer gets attached to the bottom wall and it begins to recover the attached turbulent boundary layer structure. Similar observations can be made from figure 6(a). The wall pressure coefficient shows a favorable pressure gradient on the surface upstream of the ramp followed by a region of uniform value on the ramp where the flow is separated. At $x / H_{\text {ramp }}=2.1$ we observe a pressure spike due to the stagnation conditions at the bottom ramp edge. A strong adverse pressure gradient is present at $x / H_{\text {ramp }}=2.1$ due to flow stagnation near the ramp bottom corner. The flow gets attached to the bottom wall at $x / H_{\text {ramp }}=5$. This point is marked by a positive $C_{f}$ in figure $5(\mathrm{a})$ and 


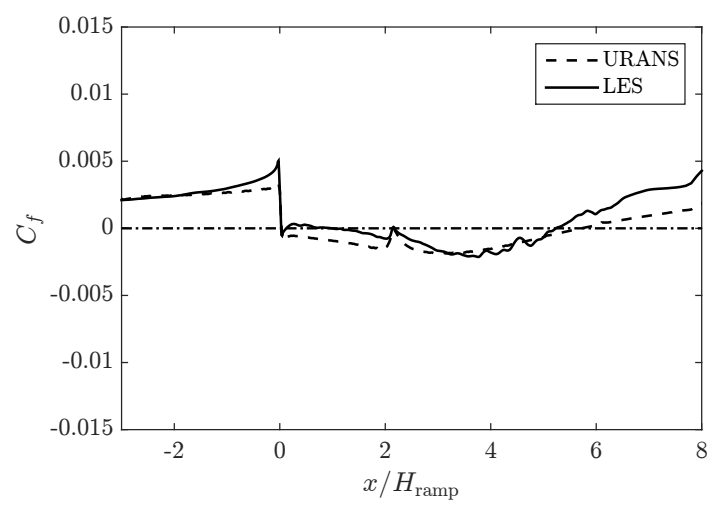

(a) without VG

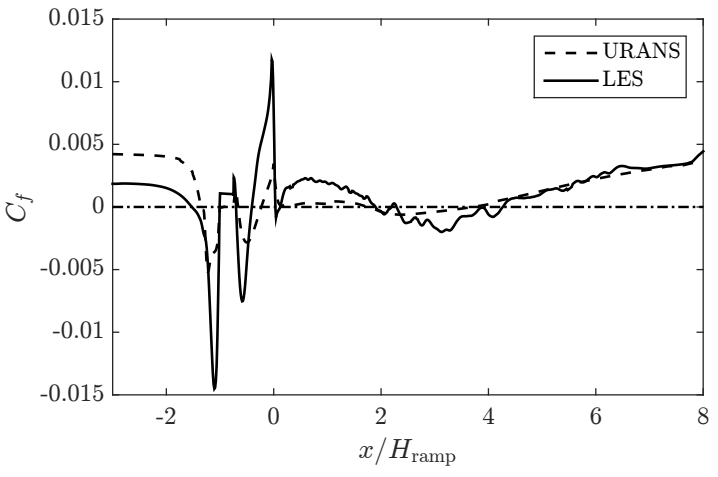

(b) with VG

Figure 5. Skin friction along the bottomwall in the plane of symmetry.

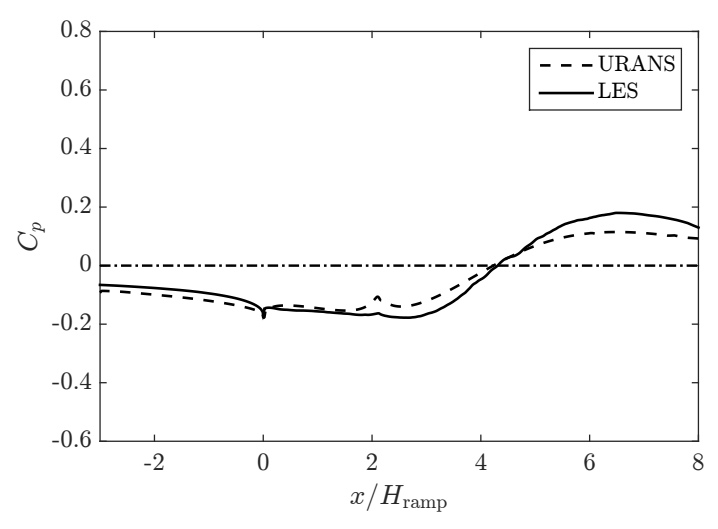

(a) without VG

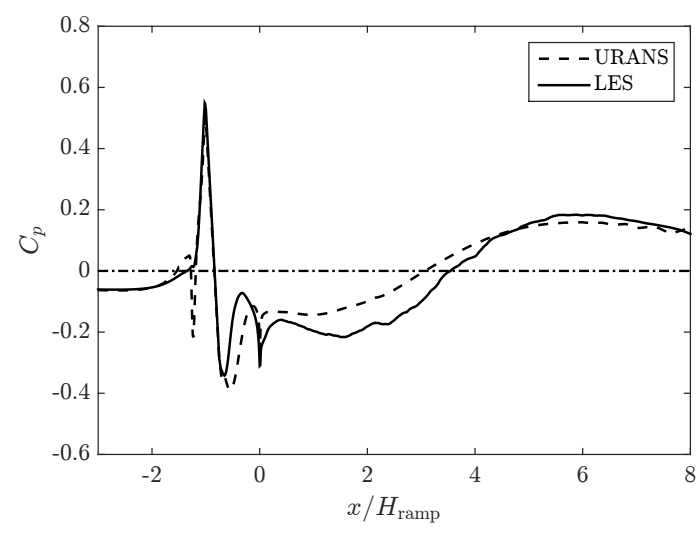

(b) with VG

Figure 6. Wall pressure co-efficient along the bottomwall in the plane of symmetry.

a favorable pressure gradient in figure 6 (a). Beyond this point the boundary layer remains attached to the bottom wall and begins to recover. URANS simulations predict the reattachment length reasonably well as compared to the LES calculations.

In the presence of the VG at $x / H_{\text {ramp }}=-0.75$ a large fluctuation in $C_{f}$ and $C_{p}$ can be seen in the vicinity of the cube from figures $5(\mathrm{~b})$ and $6(\mathrm{~b})$. Before the flow encounters the cube the URANS overpredicts the skin friction on the upstream surface on the ramp as seen in figure 5(b). The stagnation of flow in front of the cube and recirculation near the back of the cube causes negative skin friction. It also leads to high stagnation pressure in front of the cube and a low pressure region near the back surface as seen in figure 6 (b). On the ramp surface the flow is attached as suggested by a positive $C_{f}$ and constant wall pressure distribution as seen in figures 5(b) and 6(b) respectively. Near the bottom ramp corner a small value of negative skin friction coefficient suggests some reverse flow but it has a smaller magnitude as compared to the case with no VG. The flow attaches to the surface at $x / H_{\text {ramp }}=4$. The attachment length is reduced by $1 H_{\text {ramp }}$ with the use a passive VG. The URANS predicts the overall variation of $C_{f}$ and $C_{p}$ reasonably well but fails to capture the right magnitude in the separation region. This discrepancy could be because of the inadequacy of URANS to model the separated flow and the lack of adequate Reynolds stresses inside the boundary layer on the surface upstream of the ramp. We will verify this hypothesis by using a steady turbulent inflow boundary condition in URANS simulations.

Figures 7 and 8 reveal similar information about recirculation and reattachment of the flow. With no VG on the upstream surface, the flow is attached to the bottom wall. The flow separates from the ramp edge and a large recirculation of flow can be seen on the ramp surface in figures $7(\mathrm{a}), 8(\mathrm{a})$ and $8(\mathrm{~b})$. The flow attaches to the bottom wall at $x / H_{\text {ramp }}=5$. From the x-component of streamwise mean velocity profile 


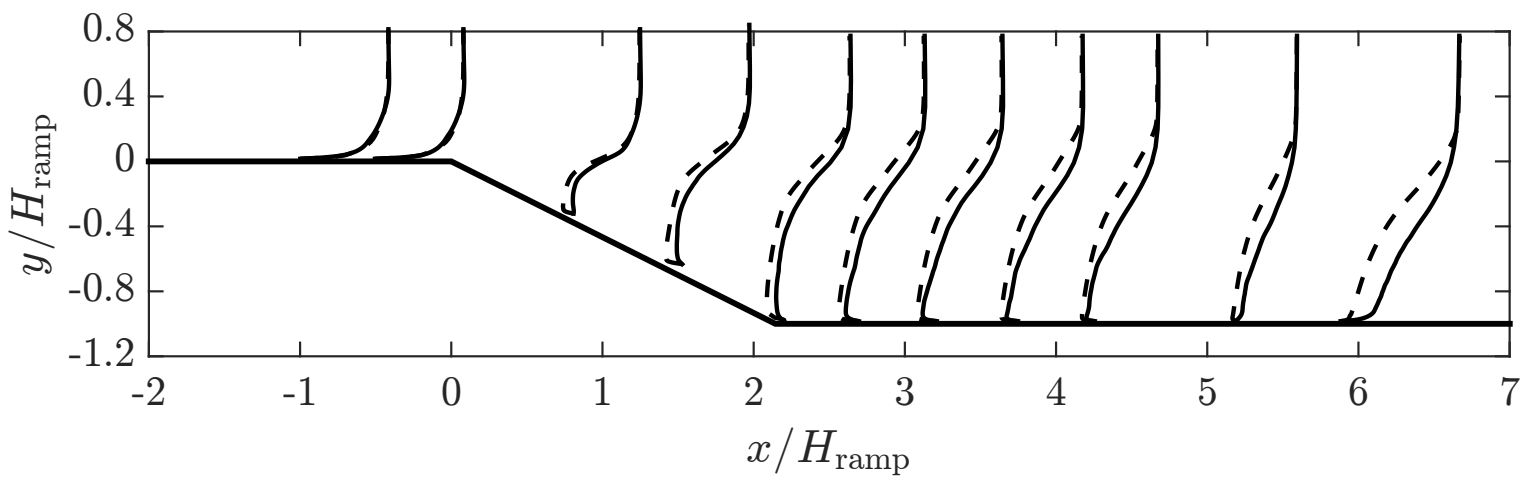

(a) without VG

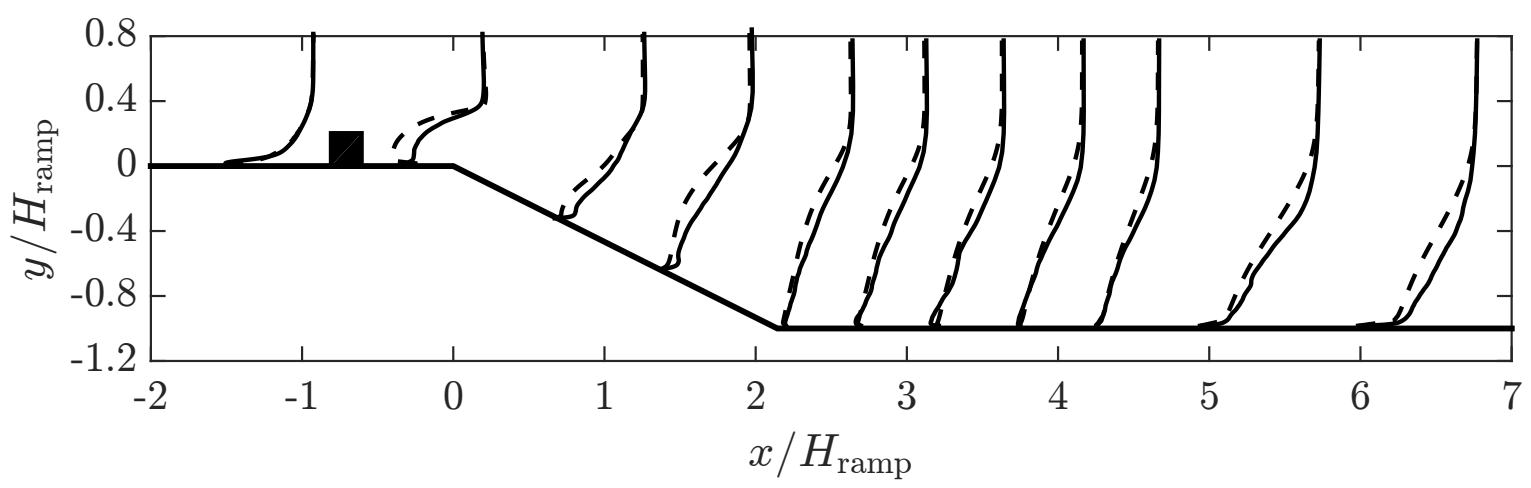

(b) with VG

Figure 7. x-component mean velocity profiles at different streamwise locations. — LES, - - - URANS.

at $x / H_{\text {ramp }}=6$ in figure $7\left(\right.$ a) we observe an inflection point at $y / H_{\text {ramp }}=-0.6$ in both LES and URANS results. This suggests that the downstream length of the domain is not long enough for the velocity to recover completely. After the flow separates from the ramp edge the LES results have a higher magnitude than corresponding URANS profiles which we think is due to higher levels of turbulence in the LES results on account of the inlet condition.

As seen from figures 7(b), 8(c) and 8(d) the streamwise velocity is highly modified in the presence of the VG. On the top surface of the ramp the recirculation of flow behind the cube entrains streamlines closer to the ramp surface. The velocity profiles on the ramp in figure $7(\mathrm{~b})$ are attached to the surface with a clear inflection point. The flow attaches to the bottom wall at $x / H_{\text {ramp }}=4$ and the boundary layer recovers downstream from this point. The LES velocity profile at $x / H_{\text {ramp }}=6$ resembles to a turbulent flat plate boundary layer. Comparing the x-component of mean streamwise velocity profiles without the VG in figure $7(\mathrm{a})$ and with VG in figure $7(\mathrm{~b})$ near $x / H_{\text {ramp }}=6$ we can conclude that the boundary layer has recovered to a greater extent in the presence of the cube.

Figure 8 describes the variation of x-component of mean velocity in the symmetry plane, with and without the VG. Without the VG we observe a larger recirculation bubble at the bottom edge of the ramp in figures $8(\mathrm{a})$ and $8(\mathrm{~b})$. The center of the recirculation bubble in LES in figure 8(a) is closer to the bottom wall as compared to the URANS prediction in figure $8(\mathrm{~b})$. We believe this discrepany is because of the inability of the $k-\omega$ SST to model the Reynolds stress in the separation region. In the presence of the VG in figures $8(\mathrm{a})$ and 8 (b) we observe higher magnitude of negative velocity behind the cube with URANS. The flow is attached on the ramp surface with a smaller recirculation bubble near the bottom edge of the ramp as compared to the case without VG.

In figure 9 we observe the variation in the turbulent kinetic energy (TKE) in the center plane of the domain, with and without a VG. In the case without VG in figures 9(a) and 9(b) a large increase in the TKE is observed downstream from the ramp edge in the separated shear layer. This peak in TKE is above 


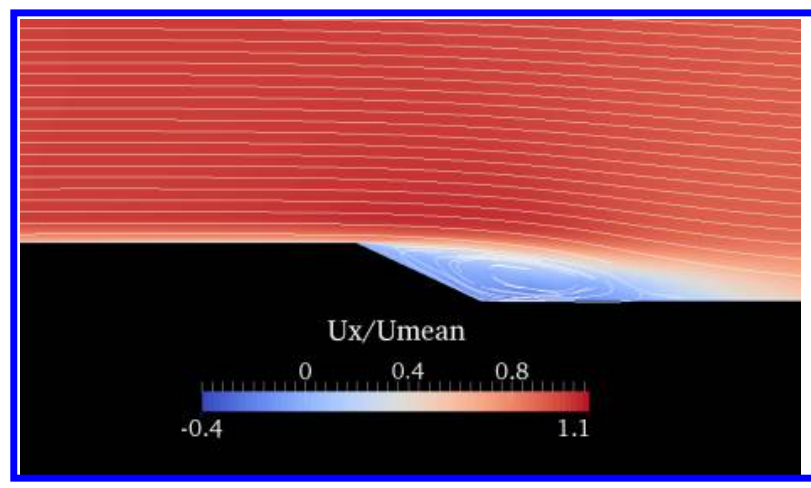

(a) LES without VG

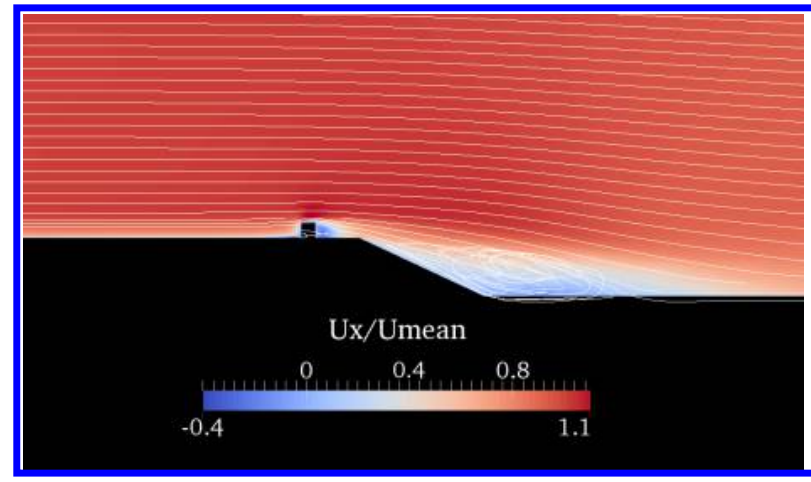

(c) LES with VG

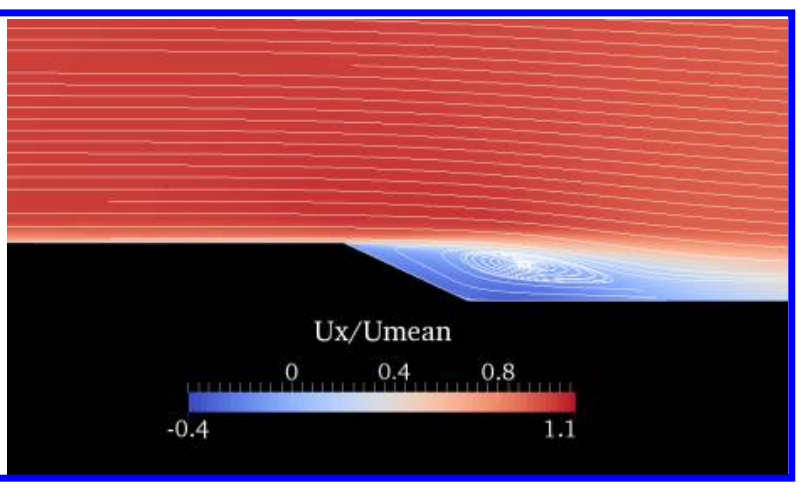

(b) URANS without VG

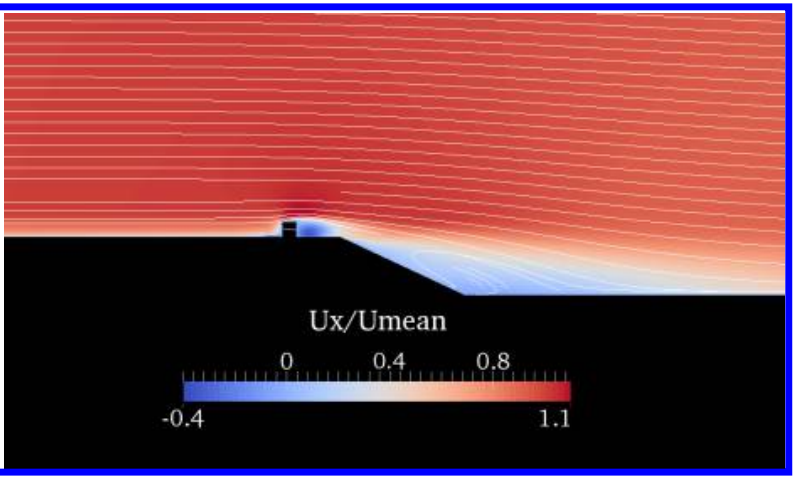

(d) URANS with VG

Figure 8. Contours of $x$-component of mean velocity non-dimensionalized by the free stream velocity plotted in the plane of symmetry.

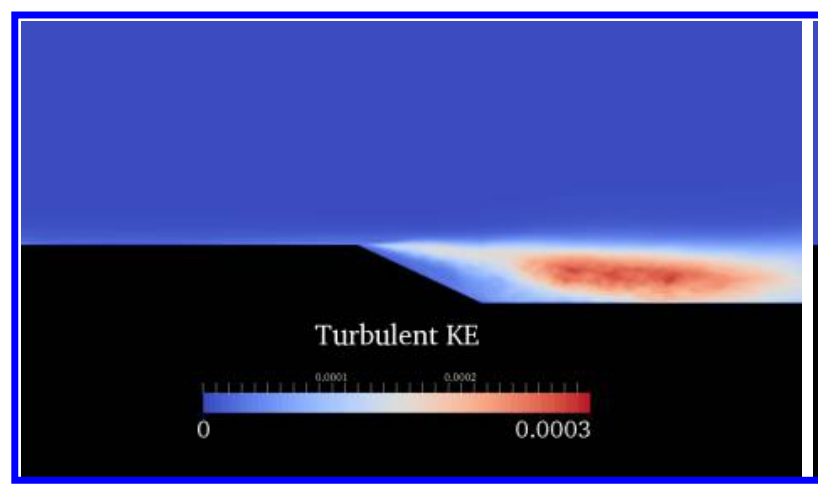

(a) LES without VG

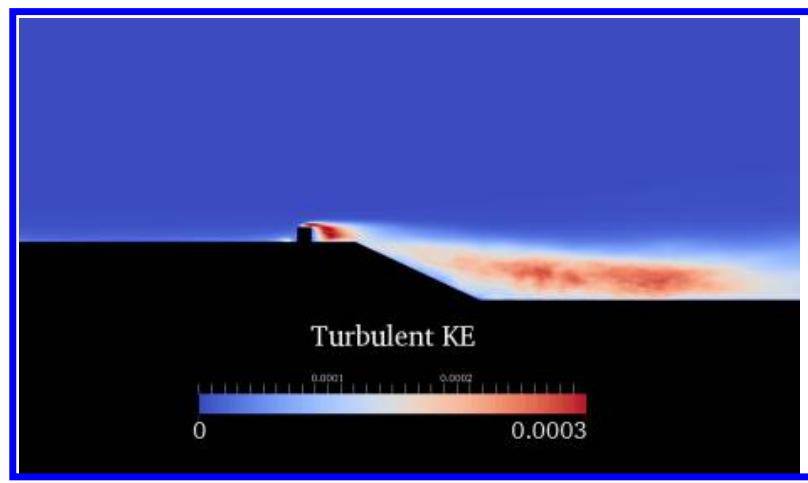

(c) LES with VG

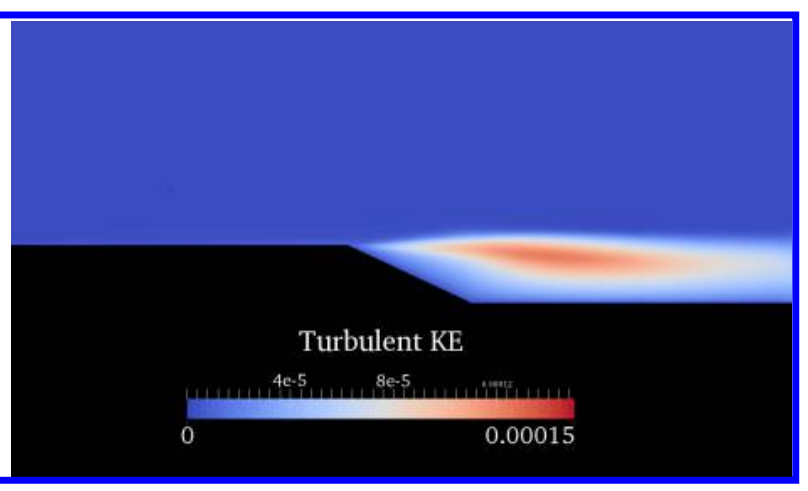

(b) URANS without VG

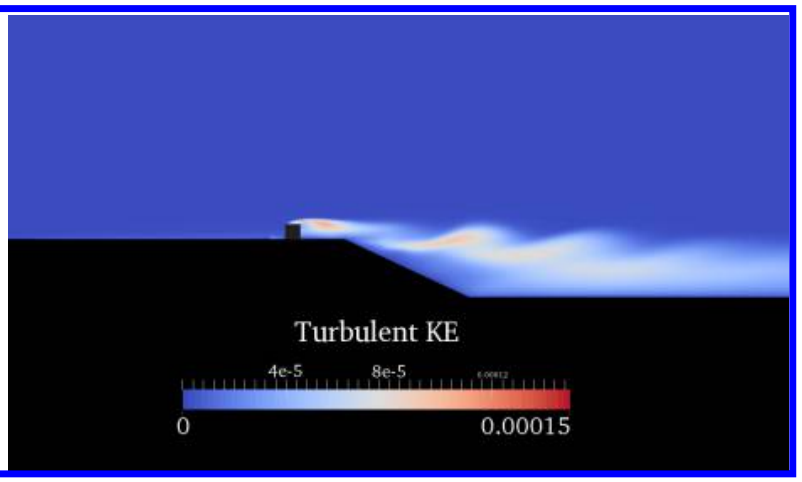

(d) URANS with VG

Figure 9. Turbulent kinetic energy contours in the plane of symmetry non-dimensionalized by free stream kinetic energy $\left(1 / 2 \rho U_{\infty}^{2}\right)$ 
the separation bubble formed near the bottom edge of the ramp. We clearly observe higher levels of TKE in LES. The separation induced KE slowly diffuses near the wall and the flow gets attached to the bottom wall. However, in the case with VG we observe from figures 9(c) and9(d) that the flow is perturbed by the VG upstream of the separation line. On account of the TKE produced at the VG a smaller separation bubble is formed at the bottom edge of the ramp. The reattachment length is reduced in this case. From the LES contours seen in figure 9(c) we observe more turbulent kinetic energy close to the wall on the ramp and the bottom surface as compared to URANS in figure 9 (d). This can be explained by observing lower magnitude of Reynolds stresses in the separation region in URANS calculations as described in section III.B.

\section{III.B. Turbulence statistics}

The streamwise and wall normal Reynolds stresses are plotted at different streamwise locations in figure 10 and figure 11. On the surface upstream of the ramp the URANS calculations have negligible turbulence near the wall. This is because of the lack of Reynolds stresses at the inflow boundary in URANS simulations. Whereas in the LES results we observe Reynolds stresses in the near wall region on the upstream ramp surface. The symbol "- represents time averaging of the Reynolds stress component. As expected both $\overline{u^{\prime 2}}$ and $\overline{v^{\prime 2}}$ have a higher magnitude in the separated region as compared to the upstream surface. The higher magnitude of Reynolds stresses downstream from the VG manifests as higher turbulent kinetic energy near the wall in the separation region.
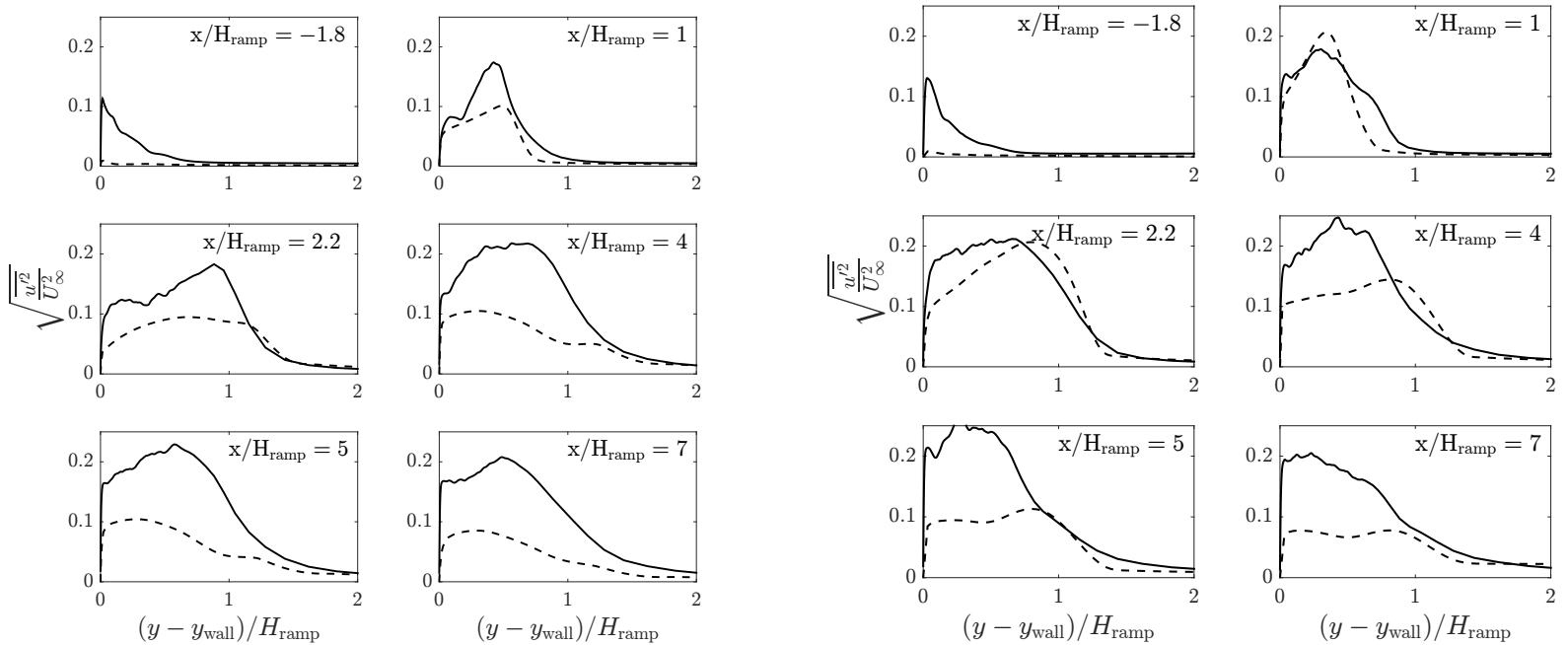

(a) without VG

(b) with VG

Figure 10. Streamwise variance $\sqrt{\frac{\overline{u^{\prime 2}}}{U_{\infty}^{2}}}$ in the center plane. — LES, - - URANS.

On the upstream surface of the ramp the boundary layer is attached to the bottom wall and the variation of the streamwise Reynolds stress $\left(\overline{u^{\prime 2}}\right)$ resembles that of a flat plate turbulent boundary layer in figures 10 (a) and $10(\mathrm{~b})$ at $x / H_{\text {ramp }}=-1.8$. In the separation region at locations $x / H_{\text {ramp }}=1,2.2,4$ and 5 in figures 10(a) and 10(b) we observe two basic characteristics observed in separated shear layer flows. One, the boundary layer thickness is increased. Two, higher Reynolds stress in the shear layer than that observed closer to the wall. Now if we closely observe the magnitude of $\overline{u^{\prime 2}}$ next to the wall in figures 10 (a) and 10(b) we can conclude that in the presence of the VG the streamwise Reynolds stress is higher close to the wall than that observed without the VG. At the last streamwise location $\left(x / H_{\text {ramp }}=7\right)$ the magnitude of $\overline{u^{\prime 2}}$ closer to the wall is higher than that in the shear layer in figure 10(b). Also the variation of $\overline{u^{\prime 2}}$ resembles that inside a flat plate turbulent boundary layer. This is because the separated boundary layer is attached earlier in the presence of the VG and has recovered to a greater extent as compared to the case without VG in figure 10(a).

The flow reattaches at $x / H_{\text {ramp }}=5$ without VG and $x / H_{\text {ramp }}=4$ with VG as seen from the streamwise variation of skin friction in figure 5. Now, if we look at the magnitude of $\overline{u^{\prime 2}}$ close to the wall after the flow reattaches in figure 10 we notice that the value remains nearly constant. However, the magnitude of $\overline{u^{\prime 2}}$ in 
the outer layer is monotonically decreasing. This suggests that the rate of recovery is different in the near layer and outer layer. The inner layer recovers faster as compared to the outer layer. Similar observations were made on a $2 \mathrm{D}$ backward-facing smooth ramp by Wasistho et al. ${ }^{26}$ In both cases without and with VG the URANS calculations underpredict the magnitude of streamwise Reynolds stress. We think this is because of the lack of near wall turbulence prior to separation in the URANS simulations.
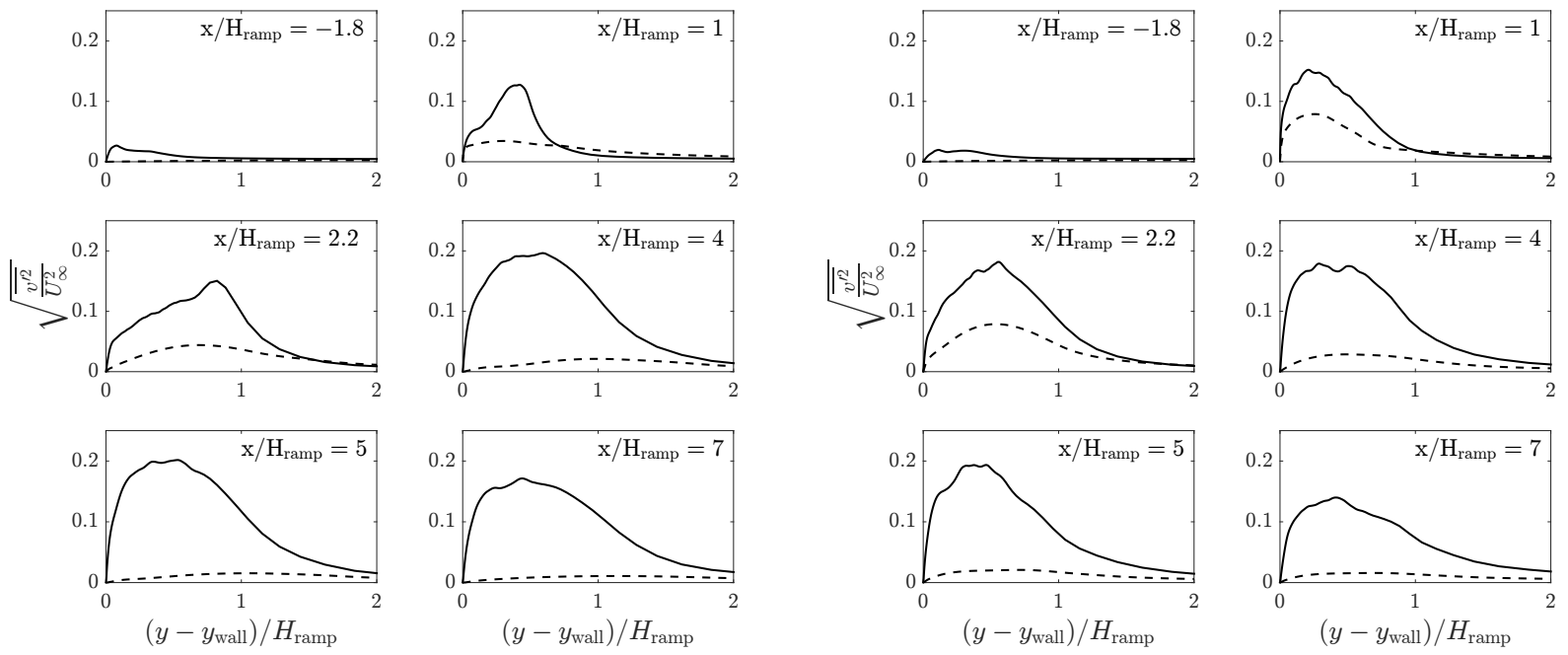

(a) without VG

(b) with VG

Figure 11. Wall normal variance $\sqrt{\frac{\overline{v^{\prime 2}}}{U_{\infty}^{2}}}$ in the center plane. —_ LES, - - URANS.

The variation of the wall normal Reynolds stress observed without the VG in figure 11(a) is similar to the the behaviour of the streamwise component observed in figure 10(a). Higher magnitude of $\overline{v^{\prime 2}}$ in the separated shear layer moves closer to the wall as the boundary layer attaches and recovers. However, in the presence of the cube we observe a different pattern. In figure 11(b) we observe that higher magnitude of $\overline{v^{\prime 2}}$ moves from close to the wall at $x / H_{\text {ramp }}=1$ to away from the wall $x / H_{\text {ramp }}=2.2$. At later streamwise locations the peak magnitude of $\overline{v^{\prime 2}}$ remains the same but the profile begins to smoothen as the boundary layer recovers. The wall normal stress levels slowly decay across the shear layer exhibiting a strong effect of flow separation. The inner layer recovery observed in $\overline{u^{\prime 2}}$ is not seen in $\overline{v^{\prime 2}}$, possibly because the peak of the wall normal Reynolds stress is located away from the wall. The URANS calculations do a poor job is capturing the variation of the wall normal Reynolds stress in cases without and with the VG.

The presence of the cube induces Reynolds stresses close to the wall in the center plane. Two important observations that can be made from the Reynolds stress profiles are that, the recovery of the Reynolds stresses is much slower than the mean boundary layer profile and the peak magnitude of the Reynolds stress profiles lie in the region where the velocity profiles experience inflection. Similar observations have been made of separated flows over a backward-facing step. These higher magnitudes of Reynolds stresses manifest as higher levels of turbulent kinetic energy (TKE) close to the wall in the separated region as seen in figure 9. The entrainment of TKE from the mean flow to the near wall region by the VG reduces the reattachment length from $5 H_{\text {ramp }}$ to $4 H_{\text {ramp }}$.

\section{III.C. Proper orthogonal decomposition (POD) analysis}

Proper Orthogonal Decomposition (POD) of unsteady flow fields help us identify the dominant modes in the flow based on their energy content. ${ }^{15,18}$ Two-dimensional separated and reattaching flows are known to exhibit two dominant characteristics namely, the vertical flapping motion of the separated shear layer and large scale vortices formed in it., ${ }^{41}$ We perform POD analysis of LES data to identify dominant modes in the separation region and investigate the effect of VG on these modes. POD consists of expressing the instantaneous flow field $u(\mathbf{x}, t)$ as a function of the mean velocity $\overline{u(\mathbf{x})}$ and the fluctuating component $u^{\prime}(\mathbf{x}, t)$. The fluctuating component $u^{\prime}(\mathbf{x}, t)$ is approximated as function of orthogonal vectors $\phi_{i}(\mathbf{x})$. 


$$
u(\mathbf{x}, t)=\overline{u(\mathbf{x})}+\sum_{i=1}^{N_{t}} a_{i}(t) \phi_{i}(\mathbf{x})
$$

In equation $1, \phi_{i}(\mathbf{x})$ refers to the $i^{t h}$ POD mode. The POD modes are arranged in descending order of energy based on the magnitude of their corresponding eigenvalues. Therefore, the first mode captures most energy of the flow, second mode captures second most energy amongst all modes and so on. This is evident from figure 12. In our POD analysis we used 500 snapshots gathered from LES in the center plane of the domain in cases with and without the VG. The snapshots were captured at a sampling frequency of

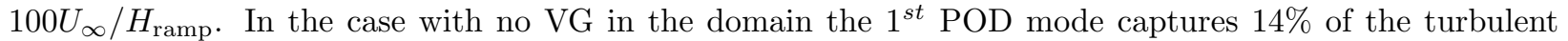
kinetic energy (TKE), the $2^{\text {nd }}$ mode captures close to $10 \%$, while the 3 rd mode captures $8 \%$ of the kinetic energy. This trend in the variation of TKE is similar to that observed by Kourta et al. ${ }^{12}$ for flow over a $25^{\circ}$ backward-facing ramp at a Reynolds number of $R e_{H}=1.85 \times 10^{5}$. In the presence of the VG the $1^{\text {st }}$ mode contains $17 \%$ of TKE whereas as the $2^{\text {nd }}$ and the $3^{\text {rd }}$ modes have values similar to the case with no VG. We believe the higher value of TKE in the $1^{\text {st }}$ mode in the case with VG is due to more TKE in the snapshots introduced by the VG.

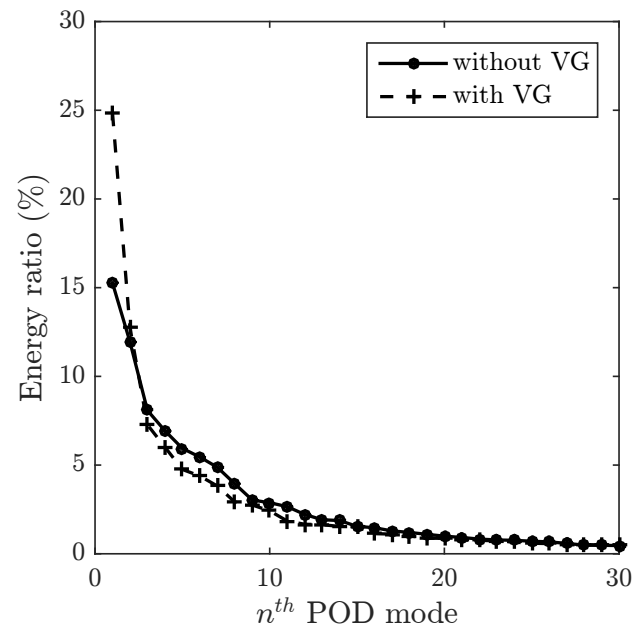

Figure 12. Energy content in POD modes.

The alternating positive and negative patches observed in the POD modes in figures 13 and 14 are not as distinct as those observed by Kourta et al. ${ }^{12}$ in their analysis. We think this is because a much higher sampling frequency used in our analysis. Even then we observe that the number of alternating patches increase with increasing modes for cases with and without the VG for both the components. If we define a wavelength as the distance between the centers of two similar patches then from figure 13 , the $1^{\text {st }}$ mode has a wavelength $4 H_{\mathrm{ramp}}$, the $2^{\text {nd }}$ mode has a wavelength of $2 H_{\mathrm{ramp}}$ while the $3^{\text {rd }}$ mode has a wavelength of $1 H_{\text {ramp. }}$.

In the POD modes of y-component of velocity in figure 14 we observe similar behavior as the $\mathrm{x}$-component. The wavelength of the modes decreases as the mode number increases. However with a close look at the modes without the VG and with VG in figure 14 we observe that with the VG, positive and negative patches are closer to the wall with a distinct negative patch approximately on the middle of the ramp. This phenomenon is not seen in the $\mathrm{x}$-component modes in figure 13. This observation is also in agreement with the fact that wall-normal Reynolds stresses diffuse from close to the wall to away from the wall in the presence of the VG. We are unable to capture the dominant flapping motion of the shear layer in the case with no VG reported by Cherry et al. ${ }^{4}$ and Kiya et al. ${ }^{11}$ for separated shear layers. We think a smaller sampling frequency will help us capture the dominant flapping mode in the separated shear layer. We can however capture the large scale vortical structures in higher POD modes (mode 2,3) as excepted. 


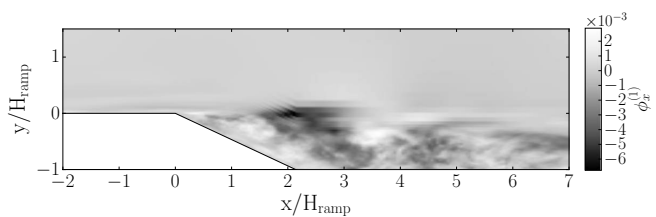

(a) $1^{\text {st }}$ mode without VG

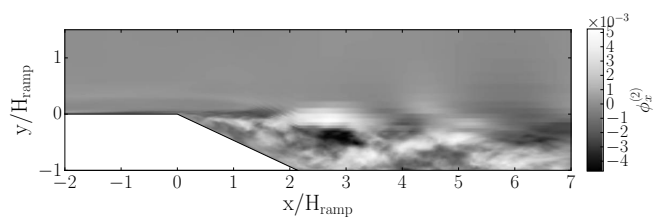

(c) $2^{\text {nd }}$ mode without $\mathrm{VG}$

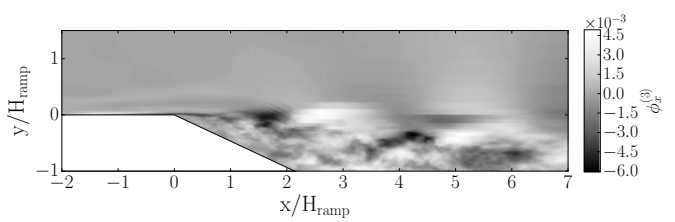

(e) $3^{r d}$ mode without $\mathrm{VG}$

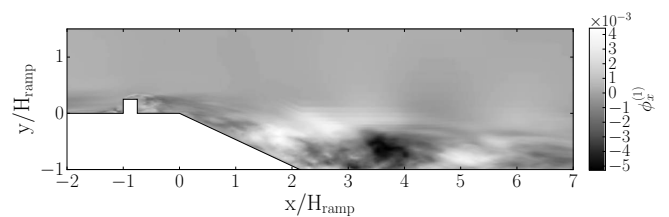

(b) $1^{\text {st }}$ mode with VG

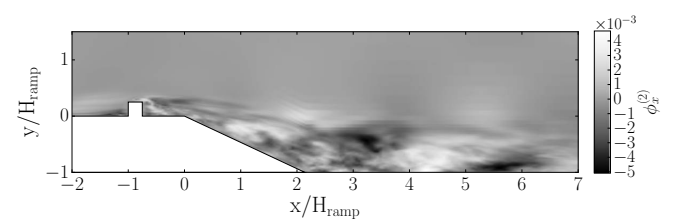

(d) $2^{\text {nd }}$ mode with VG

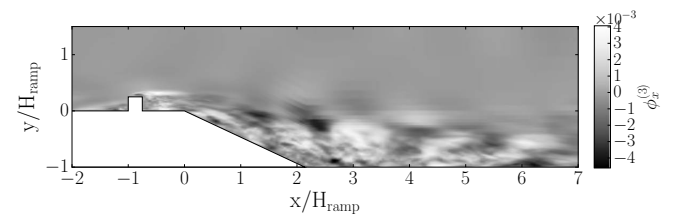

(f) $3^{\text {rd }}$ mode with VG

Figure 13. POD modes of $\mathrm{x}$-component of velocity.

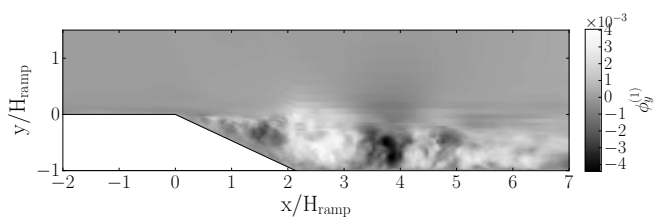

(a) $1^{\text {st }}$ mode without VG

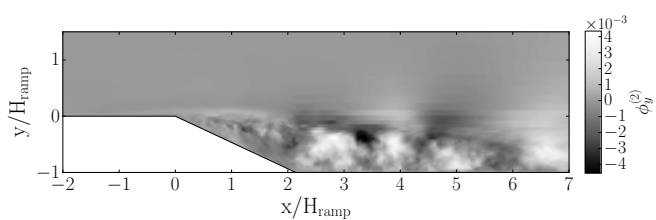

(c) $2^{\text {nd }}$ mode without VG

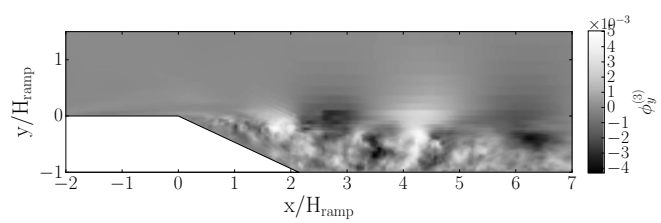

(e) $3^{\text {rd }}$ mode without VG

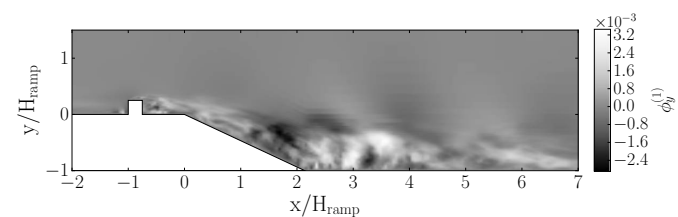

(b) $1^{\text {st }}$ mode with VG

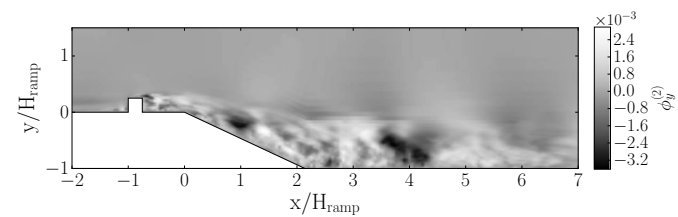

(d) $2^{\text {nd }}$ mode with VG

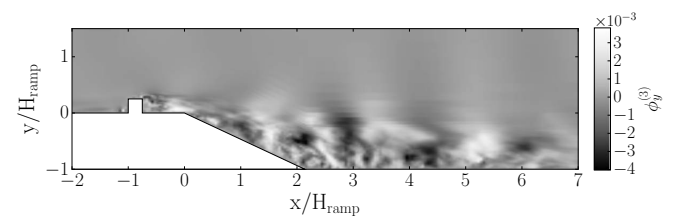

(f) $3^{\text {rd }}$ mode with VG

Figure 14. POD modes of y-component of velocity. 


\section{Conclusions}

In this study LES and URANS calculations of flow separation on a $25^{\circ}$ backward-facing ramp with and without a VG $(h \sim 0.6 \delta)$ is performed. The LES simulations are wall resolved with a grid spacing $\Delta_{y}^{+} \sim 1$. The results are considered as baseline for comparing the accuracy of the $k-\omega$ SST model for URANS calculations. A synthetic inflow condition was used as a inlet boundary condition for LES to replicate a spatially evolving turbulent boundary layer on the upstream surface of the ramp whereas as a mean turbulent inflow was used for URANS simulations. Comparing the URANS and LES results indicates that the URANS calculations do a decent job in predicting mean quantities like skin friction on the bottom wall and the reattachment length. However, they fail to capture the magnitude of mean velocity and turbulence stresses in the separated region.

From the LES simulations the reattachment length on the downstream surface was found to be $x / H_{\text {ramp }}=$ 5 with no VG and $x / H_{\text {ramp }}=4$ in the presence of the VG. A reduction of $1 H_{\text {ramp }}$ in reattachment length was obtained using a passive VG of height $h=0.64 \delta_{o}$. URANS simulations predict the reattachment length in both the cases. We believe the discrepancy in the URANS predictions could be due to the lack of turbulence inside the boundary layer prior to separation augmented by the inadequacy of $k-\omega$ SST model to capture Reynolds stresses in the separated region. We plan to verify this hypothesis by performing URANS calculations with a steady turbulent boundary layer inflow condition.

The VG helps in the reattachment process by entraining turbulent kinetic energy (TKE) from the mean flow to the near the wall region. Higher magnitudes of streamwise $\left(\overline{u^{\prime 2}}\right)$ and wall normal Reynolds stress $\left(\overline{v^{\prime 2}}\right)$ close to the wall manifests as TKE entrained from the mean flow to the near wall region. This helps to reduce the separation region and expedite the boundary layer recovery process. The inner layer recovers at a faster rate as compared to the outer layer as indicated by the streamwise Reynolds stress profiles at different streamwise locations. The recovery of Reynolds stresses in the outer layer is much slower than that of the boundary layer itself.

The POD analysis reveals the need of a smaller sampling frequency in our future study. However, we do capture the large vortical structures in the separated shear layer. Also the POD modes for y-component of velocity move closer to the wall in the presence of a VG which is consistent with our deductions from the Reynolds stresses. We plan to use the POD modes for reduced ordered modeling in future.

\section{Acknowledgements}

The authors are grateful to Dr. Pooya Movahed for his contribution in developing the turbulent inflow boundary condition. The authors would also like to thank Dr. Grzegorz P. Filip and Dr. Marc Woolliscroft for their valuable inputs in running the simulations. This work used the Extreme Science and Engineering Discovery Environment (XSEDE), which is supported by National Science Foundation grant number ACI1053575.

\section{References}

${ }^{1}$ Amy E Alving and HH Fernholz. Turbulence measurements around a mild separation bubble and downstream of reattachment. Journal of Fluid Mechanics, 322:297-328, 1996.

$>^{2}$ AC Brown, HF Nawrocki, and PN Paley. Subsonic diffusers designed integrally with vortex generators. Journal of Aircraft, 5(3):221-229, 1968.

${ }^{3}$ W Calarese and WP Crisler. AIM-85-0354 Afterbody drag reduction by vortex generators. 1985.

$\checkmark{ }^{4}$ NJ Cherry, R Hillier, and MEM Latour. Unsteady measurements in a separated and reattaching flow. Journal of Fluid Mechanics, 144(1):13, 1984.

${ }^{5}$ Julien Dandois, Eric Garnier, and Pierre Sagaut. Numerical simulation of active separation control by a synthetic jet. Journal of Fluid Mechanics, 574:25-58, 2007.

${ }^{6}$ Massimo Germano, Ugo Piomelli, Parviz Moin, and William H Cabot. A dynamic subgrid-scale eddy viscosity model. Physics of Fluids A: Fluid Dynamics (1989-1993), 3(7):1760-1765, 1991.

${ }^{7}$ Jong-Yeon Hwang and Kyung-Soo Yang. Numerical study of vortical structures around a wall-mounted cubic obstacle in channel flow. Physics of Fluids (1994-present), 16(7):2382-2394, 2004.

${ }^{8}$ Eric Johnsen, Johan Larsson, Ankit V Bhagatwala, William H Cabot, Parviz Moin, Britton J Olson, Pradeep S Rawat, Santhosh K Shankar, Björn Sjögreen, HC Yee, et al. Assessment of high-resolution methods for numerical simulations of compressible turbulence with shock waves. Journal of Computational Physics, 229(4):1213-1237, 2010.

$>{ }^{9} \mathrm{M}$ Kerho, S Hutcherson, RF Blackwelder, and RH Liebeck. Vortex generators used to control laminar separation bubbles. Journal of Aircraft, 30(3):315-319, 1993. 
${ }^{10}$ Won-Wook Kim and Suresh Menon. A new dynamic one-equation subgrid-scale model for large eddy simulations. In AIAA, Aerospace Sciences Meeting and Exhibit, 33 rd, Reno, NV, 1995.

${ }^{11}$ Masaru Kiya and Kyuro Sasaki. Structure of large-scale vortices and unsteady reverse flow in the reattaching zone of a turbulent separation bubble. Journal of Fluid Mechanics, 154:463-491, 1985.

${ }^{12}$ Azeddine Kourta, Adrien Thacker, and Romain Joussot. Analysis and characterization of ramp flow separation. Experiments in Fluids, 56(5):1-14, 2015.

${ }^{13}$ Siniša Krajnovic and Lars Davidson. Large-eddy simulation of the flow around a bluff body. AIAA journal, 40(5):927-936, 2002 .

${ }^{14}$ Hung Le. Direct numerical simulation of turbulent flow over a backward facing step. PhD thesis, Stanford University, 1995.

${ }^{15}$ YC Liang, HP Lee, SP Lim, WZ Lin, KH Lee, and CG Wu. Proper orthogonal decomposition and its applications-part i: Theory. Journal of Sound and Vibration, 252(3):527-544, 2002.

${ }^{16}$ JC Lin, FG Howard, DM Bushnell, and GV Selby. Investigation of several passive and active methods for turbulent flow separation control. In AIAA, Fluid Dynamics, 21st Plasma Dynamics and Lasers Conference, 21st, Seattle, WA, June 18-20, 1990. $17 \mathrm{p}$., volume $1,1990$.

${ }^{17}$ John C Lin. Review of research on low-profile vortex generators to control boundary-layer separation. Progress in Aerospace Sciences, 38(4):389-420, 2002.

${ }^{18}$ John Leask Lumley. The structure of inhomogeneous turbulent flows. Atmospheric Turbulence and Radio Wave Propagation, pages 166-178, 1967.

${ }^{19} \mathrm{Y}$ Na and P Moin. Direct numerical simulation of a separated turbulent boundary layer. Journal of Fluid Mechanics, 374:379-405, 1998.

${ }^{20}$ DM Rao and TT Kariya. Boundary-layer submerged vortex generators for separation control-an exploratory study. AIAA paper, 3546:1988, 1988.

${ }^{21}$ Roger L Simpson, Y-T Chew, and BG Shivaprasad. The structure of a separating turbulent boundary layer. part 1. Mean flow and Reynolds stresses. Journal of Fluid Mechanics, 113:23-51, 1981.

$\checkmark 22$ AJ Smits and DH Wood. The response of turbulent boundary layers to sudden perturbations. Annual Review of Fluid Mechanics, 17(1):321-358, 1985.

${ }^{23}$ DB Spalding. A single formula for the law of the walls. Journal of Applied Mechanics, 28(3):455-458, 1961.

${ }^{24}$ BS Stratford. The prediction of separation of the turbulent boundary layer. Journal of Fluid Mechanics, 5(01):1-16, 1959.

${ }^{25} \mathrm{HD}$ Taylor. The elimination of diffuser separation by vortex generators. United Aircraft Corporation Connecticut, Research Department Report No R-4012-3, 1947.

${ }^{26} \mathrm{~B}$ Wasistho and KD Squires. Prediction of turbulent separation over a backward-facing smooth ramp. Journal of Turbulence, (6):N1, 2005.

${ }^{27}$ David C Wilcox et al. Turbulence modeling for CFD, volume 2. DCW industries La Canada, CA, 1998.

${ }^{28}$ Xiaohua $\mathrm{Wu}$ and Parviz Moin. Direct numerical simulation of turbulence in a nominally zero-pressure-gradient flat-plate boundary layer. Journal of Fluid Mechanics, 630:5-41, 2009. 
This article has been cited by:

1. Siddhesh Shinde, Eric Johnsen, Kevin J. Maki. Understanding the effect of cube size on the near wake characteristics in a turbulent boundary layer . [Citation] [PDF] [PDF Plus]

2. Suyash Tandon, Siddhesh Shinde, Kevin J. Maki, Eric Johnsen. Flow Control Using Passive Vortex Generators . [Citation] [PDF] [PDF Plus] 